

\title{
COMPARISON RESULTS OF FINITE ELEMENT METHODS FOR THE POISSON MODEL PROBLEM*
}

\author{
C. CARSTENSEN ${ }^{\dagger}$, D. PETERSEIM ${ }^{\ddagger}$, AND M. SCHEDENSACK ${ }^{\dagger}$
}

\section{Dedicated to Professor Ulrich Langer on the occasion of his 60 th birthday}

\begin{abstract}
This paper establishes the equivalence of the conforming Courant finite element method, the nonconforming Crouzeix-Raviart finite element method, and several first-order discontinuous Galerkin finite element methods in the sense that the respective energy error norms are equivalent up to generic constants and higher-order data oscillations in a Poisson model problem. The Raviart-Thomas mixed finite element method is better than the previous methods, whereas the conjecture of the converse relation is proved to be false. This paper completes the analysis of comparison initiated by Braess [Calcolo, 46 (2009), pp. 149-155]. Two numerical benchmarks illustrate the comparison theorems and the possible strict superiority of the Raviart-Thomas mixed finite element method. Applications include least-squares finite element methods, finite volume methods. and equality of approximation classes for concepts of optimality for adaptive finite element methods.
\end{abstract}

Key words. nonconforming finite element method, Crouzeix-Raviart, Raviart-Thomas, discontinuous Galerkin. finite volume. least-squares method, approximation class

AMS subject classifications. $65 \mathrm{~N} 12,65 \mathrm{~N} 30,65 \mathrm{Y} 20$

DOI. $10.1137 / 110845707$

1. Introduction. Given a bounded polygonal domain $\Omega$ in the plane and data $f \in L^{2}(\Omega)$, the Poisson model problem seeks the weak solution $u \in H^{1}(\Omega)$ of

$$
-\Delta u=f \text { in } \Omega \text { and } u=0 \text { on } \partial \Omega \text {. }
$$

This paper compares the error of popular finite element methods (FEMs) for the numerical solution of (1.1) as depicted in Figure 1.1, the conforming Courant FEM (CFEM) [20], the nonconforming Crouzeix-Raviart FEM (CR-NCFEM) [21], the mixed Raviart-Thomas FEM (RT-MFEM) [30], and several discontinuous Galerkin FEMs (DGFEM) with respective solutions $u_{\mathrm{C}}, u_{\mathrm{CR}},\left(p_{\mathrm{RT}}, u_{\mathrm{RT}}\right)$, and $u_{\mathrm{DG}}$ based on a shape-regular triangulation of $\Omega$.

As the main result (in Theorems 2.1, 2.2, and 2.3) we will show that

$$
\left\|\nabla u-p_{\mathrm{RT}}\right\|_{L^{2}(\Omega)} \lesssim\left\|\nabla_{\mathrm{NC}}\left(u-u_{\mathrm{CR}}\right)\right\|_{L^{2}(\Omega)} \approx\left\|\nabla\left(u-u_{\mathrm{C}}\right)\right\|_{L^{2}(\Omega)} \approx\left\|u-u_{\mathrm{DG}}\right\|_{\mathrm{DG}}
$$

holds up to data oscillation $\operatorname{osc}(f, \mathcal{T})$ and up to mesh-size independent generic multiplicative constants (hidden in the notation $\lesssim$ and $\approx$ ). The norm \|\|$_{\text {DG }}$ is the standard $D G$ norm defined in (2.5). It is remarkable that those comparison results do not rely on the regularity of the solution $u$.

*Received by the editors August 24, 2011; accepted for publication (in revised form) June 27, 2012; published electronically November 1, 2012. This work is supported by the DFG Research Center Matheon Berlin and by the World Class University (WCU) program through the National Research Foundation of Korea (NRF) funded by the Ministry of Education, Science, and Technology R31-2008-000-10049-0.

http://www.siam.org/journals/sinum/50-6/84570.html

${ }^{\dagger}$ Institut für Mathematik, Humboldt-Universität zu Berlin, Unter den Linden 6, D-10099 Berlin, Germany; Department of CSE. Yonsei University, Seoul, Korea (cc@math.hu-berlin.de).

${ }^{\ddagger}$ Institut für Mathematik, Humboldt-Universität zu Berlin, Unter den Linden 6, D-10099 Berlin, Germany (peterseim@math.hu-berlin.de, schedens@math.hu-berlin.de). 

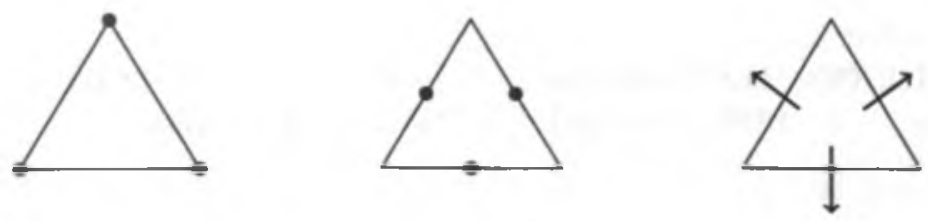

FIG. 1.1. CFEM (left), CR-NCFEM (middle), RT-MFEM (right).

A counterexample (Theorem 2.5), which is based on a sequence of domains and corresponding meshes, shows that the conjecture

$$
\left\|\nabla_{\mathrm{NC}}\left(u-u_{\mathrm{CR}}\right)\right\|_{L^{2}(\Omega)} \lesssim\left\|\nabla u-p_{\mathrm{RT}}\right\|_{L^{2}(\Omega)}
$$

is false in general. On a fixed mesh, however, the Raviart-Thomas FEM is equivalent to the other methods up to data oscillations (Theorem 2.4); the equivalence on a fixed polygonal domain independent of the mesh-size remains open.

A comparison of CFEM, CR-NCFEM, and RT-MFEM has been initiated in [7], where the hypercircle method proves $\left\|\nabla u-p_{\mathrm{RT}}\right\| \lesssim\left\|\nabla_{\mathrm{NC}}\left(u-u_{\mathrm{CR}}\right)\right\| \lesssim\left\|\nabla\left(u-u_{\mathrm{C}}\right)\right\|$. The novel result

$$
\left\|\nabla_{\mathrm{NC}}\left(u-u_{\mathrm{CR}}\right)\right\|_{L^{2}(\Omega)} \lesssim\left\|p-\Pi_{0} p\right\|_{L^{2}(\Omega)}+\operatorname{osc}(f, \mathcal{T})
$$

from $[24$, sect. 3.1$]$ with the $L^{2}$ projection of the flux $p:=\nabla u$ onto its piecewise constant integral means $\Pi_{0} p$ leads to a different proof of $\left\|\nabla_{\mathrm{NC}}\left(u-u_{\mathrm{CR}}\right)\right\|_{L^{2}(\Omega)} \lesssim$ $\left\|\nabla\left(u-u_{C}\right)\right\|_{L^{2}(\Omega)}$ with other tools. This paper gives direct proofs and a thorough comparison including DGFEM.

An immediate application to least-squares finite elenent methods improves a comparison result of [27] and disproves a further conjecture. The comparison results also clarify that various approximation classes for the optimality of adaptive FEM coincide.

The outline of this paper is as follows. Section 2 introduces the precise notation and states the main results in Theorems 2.1-2.5 and comments on it. Section 3 gives their proofs based on arguments from the a posteriori error analysis. Section 5 illustrates the equivalences in a typical situation and in the context of the counterexample of Theorem 2.5. The arguments are expected to be possibly generalized to further applications and numerical schemes as well as to higher dimensions and more general boundary conditions.

Throughout this paper, standard notation on Lebesgue and Sobolev spaces is employed and $A \lesssim B$ abbreviates an inequality $A \leq C B$ with some mesh-size independent generic constant $0 \leq C<\infty ; A \approx B$ abbreviates $A \lesssim B \lesssim A$. All hidden generic factors depend on a lower bound of the minimal angle in $\mathcal{T}$.

2. Results. This section defines the three finite element methods of Figure 1.1, introduces some class of DGFEM, and states the main results of this paper. The proofs follow in the subsequent section.

2.1. Finite element methods. Let $\mathcal{T}$ denote a shape-regular triangulation of a polygonal bounded Lipschitz domain $\Omega$ into (closed) triangles, i.e., $\bar{\Omega}=\cup_{T \in T} T$ and any two elements are either disjoint or share exactly one edge or share exactly one vertex. Let $h_{\mathcal{T}} \in P_{0}(\mathcal{T})$ denote the $\mathcal{T}$-piecewise constant mesh-size function with $\left.h_{T}\right|_{T}=\operatorname{diam}(T)$ for all $T \in \mathcal{T}$. Let $\mathcal{E}$ denote the set of edges of $\mathcal{T} ; \mathcal{E}(\Omega)$ denotes the set of interior edges, $\mathcal{E}(\partial \Omega)$ refers to the set of boundary edges. Similar notation 
applies to the vertices; $\mathcal{N}$ denotes the set of vertices, $\mathcal{N}(\Omega)$ denotes the set of interior vertices, and $\mathcal{N}(\partial \Omega)$ refers to boundary vertices. Throughout the paper, let

$P_{k}\left(\mathcal{T}: \mathbb{R}^{m}\right)=\left\{v_{k}: \Omega \rightarrow \mathbb{R}^{m} \mid\right.$ for all $T \in \mathcal{T},\left.v_{k}\right|_{T}$ is a polynomial of total degree $\left.\leq k\right\}$

denote the set of piecewise polynomials and $\Pi_{k}: L^{2}\left(\Omega ; \mathbb{R}^{m}\right) \rightarrow P_{k}\left(\mathcal{T} ; \mathbb{R}^{m}\right)$ denote the $L^{2}$ projection onto $\mathcal{T}$-piecewise polynomial functions or vectors of order $k$, e.g., $\left.\left(\Pi_{0} f\right)\right|_{T}=f_{T} f \mathrm{~d} x$ for all $T \in \mathcal{T}$ and all $f \in L^{2}\left(\Omega ; \mathbb{R}^{m}\right)$. For any interior edge $E \in \mathcal{E}(\Omega)$ there are two adjacent triangles $T^{-}$and $T^{+}$with $E=\partial T^{-} \cap \partial T^{+}$. For any $E \in \mathcal{E}(\Omega)$. let $\nu_{E}$ be the normal vector of $E$ that points from $T^{-}$to $T^{+}$, for boundary edges $E \in \mathcal{E}(\partial \Omega)$ let $\nu_{E}$ be the outward unit normal vector of $\Omega$. Define the jump of $v \in P_{k}(\mathcal{T})$ across $E \in \mathcal{E}(\Omega)$ by $[v]_{E}:=\left.v\right|_{T^{-}}-\left.v\right|_{T^{+}}$and define $[v]_{E}:=\left.v\right|_{E}$ for $E \in \mathcal{E}(\partial \Omega)$. The average of $v \in P_{k}(\mathcal{T})$ across $E \in \mathcal{E}(\Omega)$ is defined by $\langle v\rangle_{E}:=\left\langle\left. v\right|_{T^{-}}+\left.v\right|_{T^{+}}\right) / 2$ and for boundary edges $E \in \mathcal{E}(\partial \Omega)$ by $\langle v\rangle_{E}:=\left.v\right|_{E}$.

Given such a shape-regular triangulation $\mathcal{T}$, recall the FEM under consideration. CFEM. The Courant finite element space reads

$$
V_{\mathrm{C}}(\mathcal{T}):=\left\{v_{\mathrm{C}} \in P_{1}(\mathcal{T}) \mid v_{\mathrm{C}} \text { is continuous and vanishes on } \partial \Omega\right\} .
$$

The corresponding (unique) Galerkin approximation $u_{\mathrm{C}} \in V_{\mathrm{C}}(\mathcal{T})$ satisfies

$$
\int_{\Omega} \nabla u_{\mathrm{C}} \cdot \nabla v_{\mathrm{C}} \mathrm{d} x=\int_{\Omega} f v_{\mathrm{C}} \mathrm{d} x \quad \text { for all } v_{\mathrm{C}} \in V_{\mathrm{C}}(\mathcal{T})
$$

CR-NCFEM. The Crouzeix-Raviart finite element space reads

$$
\begin{aligned}
\mathrm{CR}_{0}^{1}(\mathcal{T}):=\left\{v_{\mathrm{CR}} \in P_{\mathrm{I}}(\mathcal{T}) \mid v_{\mathrm{CR}}\right. \text { is continuous at midpoints of interior } \\
\\
\text { edges and vanishes at midpoints of boundary edges }\} .
\end{aligned}
$$

A general function in $\operatorname{CR}_{0}^{1}(\mathcal{T})$ does not belong to $H^{1}(\Omega)$. However, the $\mathcal{T}$-piecewise gradient $\nabla_{\mathrm{NC}} v_{\mathrm{CR}}$, with $\left.\left(\nabla_{\mathrm{NC}} v_{\mathrm{CR}}\right)\right|_{T}=\nabla\left(v_{\mathrm{CR}} \mid \tau\right)$ for all $T \in \mathcal{T}$, exists and $\nabla_{\mathrm{NC}} v_{\mathrm{CR}} \in$ $P_{0}\left(\mathcal{T} ; \mathbb{R}^{2}\right)$. The (unique) Crouzeix-Raviart approximation $u_{\mathrm{CR}} \in \mathrm{CR}_{0}^{1}(\mathcal{T})$ satisfies

$$
\int_{\Omega} \nabla_{\mathrm{NC}} u_{\mathrm{CR}} \cdot \nabla_{\mathrm{NC}} v_{\mathrm{CR}} \mathrm{d} x=\int_{\Omega} f v_{\mathrm{CR}} \mathrm{d} x \quad \text { for all } v_{\mathrm{CR}} \in \mathrm{CR}_{0}^{1}(\mathcal{T}) .
$$

RT-MFEM. The mixed lowest-order Raviart-Thomas finite element space reads (2.3.a)

$\mathrm{RT}_{0}(\mathcal{T}):=\left\{q_{\mathrm{RT}} \in H(\operatorname{div}, \Omega) \mid\right.$ for all $T \in \mathcal{T} \exists a_{T} \in \mathbb{R}^{2} \exists b_{T} \in \mathbb{R}$ for all $x \in T$,

$$
\left.\left.q_{\mathrm{RT}}\right|_{T}(x)=a_{T}+b_{T} x\right\} \text {. }
$$

The (unique) mixed finite element approximation $\left(p_{\mathrm{RT}}, u_{\mathrm{RT}}\right) \in \mathrm{RT}_{0}(\mathcal{T}) \times B_{0}(\mathcal{T})$ satisfies

$$
\begin{gathered}
\int_{\Omega} p_{\mathrm{RT}} \cdot q_{\mathrm{RT}} \mathrm{d} x+\int_{\Omega} u_{\mathrm{RT}} \operatorname{div} q_{\mathrm{RT}} \mathrm{d} x=0 \\
\prod_{0} f+\operatorname{div} p_{\mathrm{RT}}=0 .
\end{gathered} \quad \text { for all } q_{\mathrm{RT}} \in \mathrm{RT}_{0}(\mathcal{T}) ;
$$

DGFEM. Our comparison includes some class of DGFEM which contains popular choices such as the symmetric interior penalty method (SIPG) $[22,1,25]$, the nonsymmetric interior penalty method (NIPG) [31], and the local DG (LDG) $[19,16]$. 
The abstract setting for the DGFEM under consideration is as follows. Consider the space $V_{\mathrm{DG}}(\mathcal{T}):=P_{1}(\mathcal{T})$ of $\mathcal{T}$-piecewise affines with associated jump-seminorm

$$
\left.|\cdot|\right|_{\mathfrak{J}} ^{2}:=\sum_{E \in E}|E|^{-1}\left\|[\bullet]_{E}\right\|_{L^{2}(E)}^{2}
$$

and norm

$$
\|\cdot\|_{\mathrm{DG}}:=\left(\left\|\nabla_{\mathrm{NC}} \cdot\right\|_{L^{2}(\Omega)}^{2}+\left.|\cdot|\right|_{\mathrm{J}} ^{2}\right)^{1 / 2} .
$$

The bounded and coercive (with respect to $\|\cdot\|_{\mathrm{DG}}$ ) DG bilinear form $a_{\mathrm{DG}}: V_{\mathrm{DG}}(\mathcal{T}) \times$ $V_{\mathrm{D} Z \mathrm{G}}(\mathcal{T}) \rightarrow \mathbb{R}$ extends $\left.a\right|_{V_{\mathrm{C}}(T) \times V_{\mathrm{C}}(\mathcal{T})}$ to $V_{\mathrm{DG}}(\mathcal{T}) \times V_{\mathrm{DG}}(\mathcal{T})$ and satisfies

$$
\left|a\left(v, v_{\mathrm{C}}\right)-a_{\mathrm{DG}}\left(v_{\mathrm{DG}}, v_{\mathrm{C}}\right)\right| \leq C_{1}\left\|v-v_{\mathrm{DG}}\right\|_{\mathrm{DG}}\left\|\nabla v_{\mathrm{C}}\right\|_{L^{2}(\Omega)}
$$

for all $v_{\mathrm{C}} \in V_{\mathrm{C}}(\mathcal{T}), v \in H_{0}^{1}(\Omega)$, and $v_{\mathrm{DG}} \in V_{\mathrm{DG}}(\mathcal{T})$ with some universal positive constant $C_{1}$ independent of $h_{\mathcal{T}}$. The (unique) DG approximation $u_{\mathrm{DG}} \in V_{\mathrm{DG}}(\mathcal{T})$ satisfies

$$
a_{\mathrm{DG}}\left(u_{\mathrm{DG}}, v_{\mathrm{DG}}\right)=\int_{\Omega} f v_{\mathrm{DG}} \mathrm{d} x \quad \text { for all } v_{\mathrm{DG}} \in V_{\mathrm{DG}}(\mathcal{T})
$$

Assume further that there exists some bounded linear operator $\mathrm{I}_{\mathrm{C}}: V_{\mathrm{DG}}(\mathcal{T}) \rightarrow V_{\mathrm{C}}(\mathcal{T})$ and some positive constant $C_{2}$ that does not depend on $h_{\tau}$ such that

$$
\left\|v_{\mathrm{DG}}-\mathrm{I}_{\mathrm{C}} v_{\mathrm{DG}}\right\|_{\mathrm{DG}} \leq C_{2}\left|v_{\mathrm{DG}}\right|_{\mathrm{J}}
$$

holds for all $v_{D G} \in V_{D G}(\mathcal{T})$.

It is shown in [24, sect. 3.2] that the DGFEM mentioned above (SIPG, NIPG, LDG) fit into this abstract framework. Moreover, the operator $\mathrm{I}_{\mathrm{C}}$ may be chosen based on averaging $[10,11,12,26]$; see (3.1) below for a precise definition.

2.2. Main results. This subsection presents the comparison results proved in section 3. The Lebesgue and Sobolev spaces $L^{2}(\Omega)$ and $H^{1}(\Omega)$ are defined as usual and we define $\|\cdot\|:=\|\cdot\|_{L^{2}(\Omega)}$ and $\operatorname{osc}(f, \mathcal{T}):=\left\|h_{T}\left(f-\Pi_{0} f\right)\right\|$.

THEOREM 2.1 (equivalence of CFEM and CR-NCFEM). It holds that

$$
\left\|\nabla u-\nabla u_{\mathrm{C}}\right\| \lesssim\left\|\nabla u-\nabla_{\mathrm{NC}} u_{\mathrm{CR}}\right\| \lesssim\left\|\nabla u-\nabla u_{\mathrm{C}}\right\|+\operatorname{osc}(f, \mathcal{T}) .
$$

Remark 1 (two possible conjectures). In the context of Theorem 2.1 and the hypercircle identities, two possible conjectures are that the conforming or the nonconforming error is controlled by the distance of these two discrete solutions up to oscillations, i.e.,

$$
\left\|\nabla u-\nabla u_{\mathrm{C}}\right\| \lesssim\left\|\nabla u_{\mathrm{C}}-\nabla_{\mathrm{NC}} u_{\mathrm{CR}}\right\|+\operatorname{osc}(f, \mathcal{T})
$$

and

$$
\left\|\nabla u-\nabla_{\mathrm{NC}} u_{\mathrm{CR}}\right\| \lesssim\left\|\nabla u_{\mathrm{C}}-\nabla_{\mathrm{NC}} u_{\mathrm{CR}}\right\|+\operatorname{osc}(f, \mathcal{T}) .
$$

The two statements are false, in general, because for $f \equiv 1$, and the criss-cross triangulation $\mathcal{T}$ of the unit square $\Omega=(0,1)^{2}$ into four congruent triangles as depicted in Figure 2.1, it holds that $u_{\mathrm{C}}=u_{\mathrm{CR}} \neq u$. 

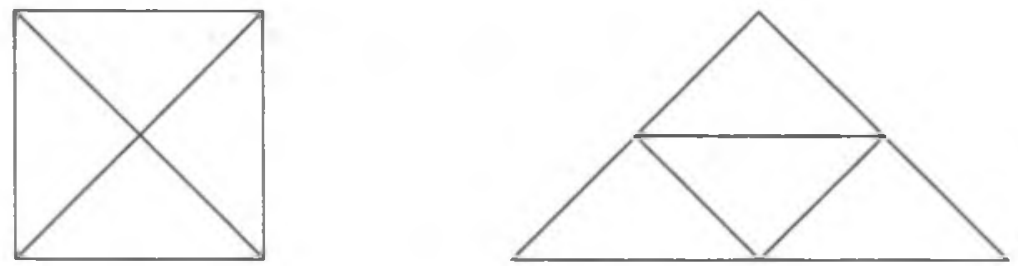

FIG. 2.1. Criss-cross triangulation of the unit square (left) and a red-refined triangle (right).

THEOREM 2.2 (equivalence of CFEM and DGFEM). It holds that

$$
\left\|\nabla u-\nabla u_{\mathrm{C}}\right\| \lesssim\left\|u-u_{\mathrm{DG}}\right\|_{\mathrm{DG}} \lesssim\left\|\nabla u-\nabla u_{\mathrm{C}}\right\|+\operatorname{osc}(f, \mathcal{T}) .
$$

Note that the first inequality in Theorem 2.1 and the first inequality in Theorem 2.2 hold without data oscillation terms.

Remark 2 (equivalence of DGFEMs). By transitivity, Theorem 2.2 establishes the equivalence (up to data oscillations) of all DGFEM under consideration, in particular SIPG, NIPG, and LDG.

THEOREM 2.3 (comparison of R'T-MFEM and CR-NCFEM). It holds that

$$
\left\|\nabla u-\nabla_{\mathrm{NC}} u_{\mathrm{CR}}\right\| \lesssim\|h f\|+\left\|\nabla u-p_{\mathrm{RT}}\right\| \lesssim\left\|\nabla u-\nabla_{\mathrm{NC}} u_{\mathrm{CR}}\right\|+\operatorname{osc}(f, \mathcal{T}) .
$$

THEOREM 2.4 (comparison of CFEM and RT-MFEM on a fixed mesh). Given any regular triangulation $\mathcal{T}$ of the polygonal Lipschitz domain $\Omega$ in $\mathbb{R}^{2}$, there exists some constant $C(\mathcal{T})$ such that

$$
\left\|\nabla\left(u-u_{\mathrm{C}}\right)\right\| \leq C(\mathcal{T})\left(\left\|\nabla u-p_{\mathrm{RT}}\right\|+\operatorname{osc}(f, \mathcal{T})\right) .
$$

The constant $C(\mathcal{T})$ may depend on the triangulation $\mathcal{T}$, but does not depend on the right-hand side $f \in L^{2}(\Omega)$ and the solution $u \in H_{0}^{1}(\Omega)$ or its regularity.

THEOREM 2.5 (superiority of RT-MFEM). The conjecture

$$
\left\|\nabla u-\nabla_{\mathrm{NC}} u_{\mathrm{CR}}\right\| \lesssim\left\|\nabla u-p_{\mathrm{RT}}\right\|+\operatorname{osc}(f . \mathcal{T})
$$

is false, in general, in the sense that, given $f \equiv 2$ and $M>0$, there exist some convex Lipschitz domain $\Omega=\Omega_{M}$ and a quasi uniform triangulation $\mathcal{T}=\mathcal{T}_{M}$ such that

$$
M\left(\left\|\nabla u-p_{\mathrm{RT}}\right\|+\operatorname{osc}(f, \mathcal{T})\right) \leq\left\|\nabla u-\nabla_{\mathrm{NC}} u_{\mathrm{CR}}\right\| .
$$

Remark 3. The counterexample in the proof of Theorem 2.5 explains that for a sequence of triangulations $\left(\mathcal{T}_{\ell}\right)_{\ell \in \mathbb{N}}$ the corresponding sequence of constants $C\left(\mathcal{T}_{\ell}\right)$ in Theorem 2.4 may be unbounded. The indirect proof of Theorem 2.4 employs compactness and, hence, does not provide further information on that growth. It is conjectured that $C(\mathcal{T})$ depends only on the polygonal domain $\Omega$ and interior angles of $\mathcal{T}$ (see section 5.1 for a numerical experiment) but not on the mesh-size.

\section{Proofs.}

3.1. Proof of Theorem 2.1. The first proposition is already included in [13, Theorem 5.1] with a different proof.

Proposition 3.1. Any $v_{\mathrm{CR}} \in \mathrm{CR}_{0}^{1}(\mathcal{T})$ satisfies

$$
\min _{v_{C} \in V_{C}(\mathcal{T})}\left\|\nabla_{\mathrm{NC}} v_{\mathrm{CR}}-\nabla v_{\mathrm{C}}\right\| \approx \min _{v \in H_{0}^{1}(\Omega)}\left\|\nabla_{\mathrm{NC}} v_{\mathrm{CR}}-\nabla v\right\| \text {. }
$$


Proof. Let $\omega_{T}:=\cup\{K \in \mathcal{T} \mid T \cap K \neq \emptyset\}$ denote the patch of first order layers around $T$ and let $\mathcal{E}\left(\omega_{T}\right):=\{E \in \mathcal{E} \mid E \cap T \neq \emptyset\}$ denote its edges. For $z \in \mathcal{N}$ define $\mathcal{T}(z):=\{T \in \mathcal{T} \mid z \in T\}$.

Given $v_{\mathrm{CR}} \in \mathrm{CR}_{0}^{1}(\mathcal{T})$ define $\mathrm{I}_{\mathrm{C}} v_{\mathrm{CR}} \in V_{\mathrm{C}}(\mathcal{T})$ by

$$
\left(\mathrm{I}_{\mathrm{C}} v_{\mathrm{CR}}\right)(z)=|\mathcal{T}(z)|^{-1} \sum_{T \in \mathcal{T}(z)}\left(v_{\mathrm{CR}} \mid T\right)(z)
$$

for any interior node $z \in \mathcal{N}(\Omega)$. For $T \in \mathcal{T}$ and $v_{\mathrm{CR}} \in \mathrm{CR}_{0}^{1}(\mathcal{T})$, define

$$
\rho_{1}\left(v_{\mathrm{CR}}\right):=\left\|\nabla_{\mathrm{NC}}\left(v_{\mathrm{CR}}-\mathrm{I}_{\mathrm{C}} v_{\mathrm{CR}}\right)\right\|_{L^{2}(T)}
$$

and

$$
\rho_{2}\left(v_{\mathrm{CR}}\right):=\sqrt{\sum_{E \in \mathcal{E}\left(\omega^{\prime}\right)}|E| \|\left[\left.\left(\nabla_{\mathrm{NC}} v_{\mathrm{CR}}\right) \cdot \tau_{E}\right|_{E} \|_{L^{2}(E)}^{2}\right.},
$$

where $\tau_{E}$ denotes a unit vector tangential to $E$. If $\rho_{2}\left(v_{\mathrm{CR}}\right)=0$, then $v_{\mathrm{CR}}$ is continuous on $\omega_{T}$ and $\left.v_{\mathrm{CR}}\right|_{E}=0$ for a boundary edge $E \subset \partial \Omega \cap \bar{\omega}_{T}$, hence $\mathrm{I}_{\mathrm{C}} v_{\mathrm{CR}}=v_{\mathrm{CR}}$ on $T$ and $\rho_{1}\left(v_{\mathrm{CR}}\right)=0$. Since $\rho_{1}$ and $\rho_{2}$ are seminorms on $\mathrm{CR}_{0}^{1}(\mathcal{T})$, there exists a constant, such that $\rho_{1} \lesssim \rho_{2}$ on $\mathrm{CR}_{0}^{1}(\mathcal{T})$. A scaling argument shows that the constant is independent of the mesh-size. The sum over all $T \in \mathcal{T}$ and the bounded overlap of the patches $\left(\omega_{T} \mid T \in \mathcal{T}\right)$ show that

$$
\begin{aligned}
\left\|\nabla_{\mathrm{NC}}\left(v_{\mathrm{CR}}-\mathrm{I}_{\mathrm{C}} v_{\mathrm{C}(\mathrm{R})}\right)\right\|^{2} & \lesssim \sum_{T \in \mathcal{T}} \sum_{E \in \mathcal{E}\left(\omega_{T}\right)}|E|\left\|\left\{\left(\nabla_{\mathrm{NC}} \cdot v_{\mathrm{CR}}\right) \cdot \tau_{E}\right]_{E}\right\|_{L^{2}(E)}^{2} \\
& \lesssim \sum_{E \in \mathcal{E}}|E|\left\|\left[\left(\nabla_{\mathrm{NC}} v_{\mathrm{CR}}\right) \cdot \tau_{E}\right]_{E}\right\|_{L^{2}(E)}^{2} .
\end{aligned}
$$

A standard argument with edge-bubble functions (cf. [32]; see [13, Proof of Theorem 5.1 for details) shows

$$
\sqrt{\sum_{E \in \mathcal{E}}|E|\left\|\left[\left(\nabla_{\mathrm{NC}} v_{\mathrm{CR}}\right) \cdot r_{E}\right]_{E}\right\|_{L^{2}(E)}^{2}} \lesssim \min _{v \in H_{3}^{1}(\Omega)}\left\|\nabla_{\mathrm{NC}} v_{\mathrm{CR}}-\nabla v\right\| .
$$

Hence, one inequality is proven. The reverse inequality follows from $\nabla V_{\mathrm{C}}(\mathcal{T}) \subset$ $\nabla H_{0}^{1}(\Omega)$.

The remaining part of this subsection is devoted to our proof of Theorem 2.1. The inclusion $V_{\mathrm{C}}(\mathcal{T}) \subset \mathrm{CR}_{0}^{1}(\mathcal{T})$ and Galerkin orthogonality show

$$
\left\|\nabla_{\mathrm{NC}} u_{\mathrm{CR}}-\nabla u_{\mathrm{C}}\right\|=\min _{v_{\mathrm{C}} \in V_{\mathrm{C}}(\mathcal{T})}\left\|\nabla_{\mathrm{NC}} u_{\mathrm{CR}}-\nabla v_{\mathrm{C}}\right\| .
$$

Together with Proposition 3.1 and the triangle inequality it follows that

$$
\left\|\nabla u-\nabla u_{C}\right\| \leq\left\|\nabla u-\nabla_{N C} u_{C R}\right\|+\left\|\nabla_{N C} u_{C R}-\nabla u_{C}\right\| \lesssim\left\|\nabla u-\nabla_{\mathrm{NC}} u_{C R}\right\|,
$$

which is the first inequality in Theorem 2.1.

A proof of the second inequality can be found in [7], while here a different, direct proof is given. 
Let $e:=\mathrm{I}_{\mathrm{NC}} u-u_{\mathrm{CR}}$, where the nonconforming interpolation $\mathrm{I}_{\mathrm{NC}} u \in \mathrm{CR}_{0}^{1}(\mathcal{T})$ is defined uniquely by

$$
f_{E} \mathrm{I}_{N C} u \mathrm{~d} s=f_{E} u \mathrm{~d} s \quad \text { for all } E \in \mathcal{E} \text {. }
$$

Since $\nabla_{\mathrm{NC}}\left(\mathbf{l}_{\mathrm{NC}} u\right)=\Pi_{0}(\nabla u)$, it holds that

$$
\begin{aligned}
\left\|\nabla u-\nabla_{\mathrm{NC}} u_{\mathrm{CR}}\right\|_{L^{2}(\Omega)} & \leq\left\|\nabla u-\nabla_{\mathrm{NC}} \mathrm{I}_{\mathrm{NC}} u\right\|_{L^{2}(\Omega)}+\left\|\nabla_{\mathrm{NC}} e\right\|_{L^{2}(\Omega)} \\
& \leq\left\|\nabla\left(u-u_{\mathrm{C}}\right)\right\|_{L^{2}(\Omega)}+\left\|\nabla_{\mathrm{NC}} e\right\|_{L^{2}(\Omega)} .
\end{aligned}
$$

Since $\nabla_{\mathrm{NC}} e$ is constant on $E \in \mathcal{E},[e]$ is affine on $E \in \mathcal{E}$ and vanishes in the midpoint of $E$, it follows for $e_{\mathrm{C}}:=\mathrm{I}_{\mathrm{C}} e \in V_{\mathrm{C}}(\mathcal{T})$ (with $\mathrm{I}_{\mathrm{C}}$ from (3.1)) that

$$
\begin{aligned}
\left\|\nabla_{\mathrm{NC}}\right\|_{L^{2}(\Omega)}^{2} & =\int_{\Omega} \nabla_{\mathrm{NC}} e \cdot \nabla_{\mathrm{NC}}\left(e-e_{\mathrm{C}}\right) \mathrm{d} x \\
& =\sum_{E \in \mathcal{E}} \int_{E}\left[\left(e-e_{\mathrm{C}}\right) \nabla_{\mathrm{N} C} e \cdot \nu_{E}\right]_{E} \mathrm{~d} s \\
& \leq \sum_{E \in \mathcal{E}(\Omega)}\left\|\left[\nabla_{\mathrm{NC}} e \cdot \nu_{E}\right]_{E}\right\|_{L^{2}(E)}\left\|\left(e-e_{\mathrm{C}}\right\rangle_{E}\right\|_{L^{2}(E)} \\
& \leq \sqrt{\sum_{E \in \mathcal{E}(\Omega)}|E|\left\|\left[\nabla_{\mathrm{NC}} e \cdot \nu_{E}\right]_{E}\right\|_{L^{2}(E)}^{2}} \sqrt{\sum_{E \in \mathcal{E}}|E|^{-1}\left\|\left\langle e-e_{C}\right\rangle_{E}\right\|_{L^{2}(E)}^{2}}
\end{aligned}
$$

Let $\Omega_{E}:=\cup\{T \in \mathcal{T} \mid E \cap T \neq \emptyset\}$ denote the patch of first order around $E$ and let $\mathcal{T}\left(\Omega_{E}\right)$ denote its triangles. Define for $v_{\mathrm{CR}} \in \mathrm{CR}^{1}\left(\mathcal{T}\left(\Omega_{E}\right)\right)$

$$
\rho_{3}\left(v_{\mathrm{CR}}\right):=|E|^{-\frac{1}{2}}\left\|\left\langle v_{\mathrm{CR}}-\mathrm{I}_{\mathrm{C}} v_{\mathrm{CR}}\right\rangle_{E}\right\|_{L^{2}(E)} \quad \text { and } \quad \rho_{4}\left(v_{\mathrm{CR}}\right):=\left\|\nabla_{\mathrm{NC}} v_{\mathrm{CR}}\right\|_{L^{2}\left(\Omega_{E}\right)} .
$$

If $\rho_{4}\left(v_{\mathrm{CR}}\right)=0$, then $v_{\mathrm{CR}}$ is constant on each $T \in \mathcal{T}\left(\Omega_{E}\right)$. Since $v_{\mathrm{CR}}$ is continuous on the midpoints of interior edges of $\mathcal{T}\left(\Omega_{E}\right), v_{\mathrm{CR}}$ is constant on $\Omega_{E}$. Hence, $v_{\mathrm{CR}}=\mathrm{I}_{\mathrm{C}} v_{\mathrm{CR}}$ on $E$ and $\rho_{3}\left(v_{\mathrm{CR}}\right)=0$. Since $\rho_{3}$ and $\rho_{4}$ are seminorms on $\mathrm{CR}^{1}\left(\mathcal{T}\left(\Omega_{E}\right)\right)$, there exists a constant such that $\rho_{3} \lesssim \rho_{4}$ on $\mathrm{CR}^{1}\left(\mathcal{T}\left(\Omega_{E}\right)\right)$. A scaling argument shows that the constant is independent of the mesh-size. The sum over all interior edges of $\mathcal{T}$ and the bounded overlap of the patches $\left(\Omega_{E} \mid E \in \mathcal{E}\right)$ show that

$$
\sum_{E \in \mathcal{E}}|E|^{-1}\left\|\left\langle e-e_{C}\right\rangle_{E}\right\|_{L^{2}(E)}^{2} \lesssim\left\|\nabla_{N C} e\right\|^{2}
$$

This leads to

$$
\left\|\nabla_{\mathrm{NC}} e\right\|^{2} \lesssim \sum_{E \in \mathcal{E}(\Omega)}|E|\left\|\left[\nabla_{\mathrm{NC}} e \cdot \nu_{E}\right]_{E}\right\|_{L^{2}(E)}^{2}
$$

For any vertex $x \in \mathcal{N}$, let $\varphi_{x}$ denote the associated hat function (i.e., $\varphi_{x}$ is continuous, $\mathcal{T}$-piecewise affine, and $\varphi_{x}(y)=\delta_{x y}$ for all $\left.y \in \mathcal{N}\right)$. Given any $E=\operatorname{conv}\{a, b\} \in \mathcal{E}$ with $E=T_{+} \cap T_{-}$for $T_{+}=\operatorname{conv}\{a, b, c\}, T_{-}=\operatorname{conv}\{a, b, d\} \in \mathcal{T}$, let $b_{E}:=6 \varphi_{a} \varphi_{b}-$ 
$10 \varphi_{a} \varphi_{b} \varphi_{c}-10 \varphi_{a} \varphi_{b} \varphi_{d}$ be some bubble function supported on $\omega_{E}:=T_{+} \cup T_{-}$. Compute

$$
\begin{aligned}
|E|^{1 / 2} & \left\|\left[\left(\nabla_{\mathrm{NC}} e\right) \cdot \nu_{E}\right]_{E}\right\|_{L^{2}(E)}=\left|\int_{E}\left[\left(\nabla_{\mathrm{NC}} \mathrm{I}_{\mathrm{NC}} u-\nabla_{\mathrm{NC}} u_{\mathrm{CR}}\right) \cdot \nu_{E}\right]_{E} \mathrm{~d} s\right| \\
= & \left|\int_{E} b_{E}\left[\left(\nabla_{\mathrm{NC}} \mathrm{I}_{\mathrm{NC}} u-u\right) \cdot \nu_{E}\right]_{E} \mathrm{~d} s-\int_{E} \psi_{E}\left[\left(\nabla_{\mathrm{NC}} u_{\mathrm{CR}}\right) \cdot \nu_{E}\right]_{E} \mathrm{~d} s\right| \\
= & \left|\int_{\omega_{E}} \nabla_{\mathrm{NC}}\left(\mathrm{I}_{\mathrm{NC}} u-u\right) \cdot \nabla b_{E} \mathrm{~d} x-\int_{\Omega} \Delta u b_{E} \mathrm{~d} x-\int_{\Omega} \nabla_{\mathrm{NC}} u_{\mathrm{CR}} \cdot \nabla_{\mathrm{NC}} \psi_{E} \mathrm{~d} x\right| \\
\leq & \int_{\Omega} \nabla_{\mathrm{NC}}\left(\mathrm{I}_{\mathrm{NC}} u-u\right) \cdot \nabla b_{E} \mathrm{~d} x|+| \int_{\Omega} f\left(b_{E}-\psi_{E}\right) \mathrm{d} x \mid .
\end{aligned}
$$

Since, by definition, $f_{T_{ \pm}}\left(b_{E}-\psi_{E}\right) \mathrm{d} x=0$ and $\left\|b_{E}-\psi_{E}\right\|_{L \times(\Omega)} \approx 1$, the Poincare inequality leads to

$$
\left|\int_{\Omega} f\left(b_{E}-\psi_{E}\right) \mathrm{d} x\right|=\left|\int_{\omega_{E}}\left(f-\Pi_{0} f\right)\left(b_{E}-\psi_{E}\right) \mathrm{d} x\right| \lesssim\left|\omega_{E}\right|^{1 / 2}\left\|f-\Pi_{0} f\right\|_{L^{2}\left(\omega_{:}\right)} .
$$

Moreover, $\left\|\nabla b_{E}\right\|_{L^{2}(\Omega)} \approx 1$ and $\nabla_{\mathrm{NC}} \mathrm{I}_{\mathrm{NC}} u=\Pi_{0}(\nabla u)$ yield

$$
\left|\int_{\Omega} \nabla_{\mathrm{NC}}\left(\mathrm{I}_{\mathrm{NC}} u-u\right) \cdot \nabla b_{E} \mathrm{~d} x\right| \lesssim\left\|\nabla_{\mathrm{NC}}\left(u-\mathrm{I}_{\mathrm{NC}} u\right)\right\|_{L^{2}\left(\omega_{k}\right)} \leq\left\|\nabla\left(u-u_{C}\right)\right\|_{L^{2}\left(\omega_{k}\right)} .
$$

The combination of (3.2)-(3.6) plus the finite overlap of $\left(\omega_{E} \mid E \in \mathcal{E}\right)$ proves the second inequality in Theorem 2.1 .

3.2. Proof of Theorem 2.2. The triangle inequality yields

$$
\left\|\nabla\left(u-u_{C}\right)\right\|=\left\|u-u_{C}\right\|_{\mathrm{DG}} \leq\left\|u-u_{\mathrm{DG}}\right\|_{\mathrm{DG}}+\left\|u_{\mathrm{DG}}-u_{\mathrm{C}}\right\|_{\mathrm{DG}} .
$$

The inclusion $V_{\mathrm{C}}(\mathcal{T}) \subset V_{\mathrm{DG}}(\mathcal{T})$, Galerkin orthogonality

$$
a_{\mathrm{DG}}\left(u_{\mathrm{DG}}-u_{\mathrm{C}}, u_{\mathrm{DG}}-u_{\mathrm{C}}\right)=\min _{v_{\mathrm{C}} \in V_{\mathrm{C}}(T)} a_{\mathrm{DG}}\left(u_{\mathrm{DG}}-u_{\mathrm{C}}, u_{\mathrm{DG}}-v_{\mathrm{C}}\right),
$$

coercivity and boundedness of $a_{\mathrm{DG}}$ (with respect to $\|\bullet\|_{\mathrm{DG}}$ ), and the property (2.8) of the averaging operator $I_{C}$ yield

$$
\begin{aligned}
\left\|u_{\mathrm{DG}}-u_{\mathrm{C}}\right\|_{\mathrm{DG}}^{2} & \lesssim a_{\mathrm{DG}}\left(u_{\mathrm{DG}}-u_{\mathrm{C}}, u_{\mathrm{DG}}-u_{\mathrm{C}}\right) \\
& =a_{\mathrm{DG}}\left(u_{\mathrm{DG}}-u_{\mathrm{C}}, u_{\mathrm{DG}}-\mathrm{I}_{\mathrm{C}} u_{\mathrm{DG}}\right) \lesssim\left\|u_{\mathrm{DG}}-u_{\mathrm{C}}\right\|_{\mathrm{DG}}\left|u_{\mathrm{DG}}\right|_{\mathrm{J}} .
\end{aligned}
$$

Since the jump seminorm vanishes on $V$, we have

$$
\left\|u_{\mathrm{DG}}-u_{\mathrm{C}}\right\|_{\mathrm{DG}} \lesssim \inf _{v \in V}\left\|u_{\mathrm{DG}}-v\right\|_{\mathrm{DG}} .
$$

The combination of (3.7)-(3.9) proves the first inequality in Theorem 2.2.

The proof of the second inequality follows directly from the novel result

$$
\left\|u-u_{\mathrm{DG}}\right\|_{\mathrm{DG}} \lesssim \min _{v_{\mathrm{DG}} \in V_{\mathrm{DG}}(\mathcal{T})}\left\|u-v_{\mathrm{DG}}\right\|_{\mathrm{DG}}+\operatorname{osc}(f, \mathcal{T})
$$

from $[24$, sect. 3.2]. 
3.3. Proof of Theorem 2.3. Let $\tilde{u}_{\mathrm{CR}} \in \mathrm{CR}_{0}^{1}(\mathcal{T})$ denote the Crouzeix-Raviart solution with respect to the right-hand side $\Pi_{0} f$. Marini [28] shows that

$$
p_{\mathrm{RT}}=\nabla_{\mathrm{NC}} \bar{u}_{\mathrm{CR}}-\frac{1}{2}\left(\Pi_{0} f\right)(\bullet-\operatorname{mid}(\mathcal{T})),
$$

where $\left.\operatorname{mid}(\mathcal{T})\right|_{T}=\operatorname{mid}(T)$ and $\operatorname{mid}(T)$ denotes the barycenter of $T \in \mathcal{T}$ and $(\bullet-$ $\operatorname{mid}(T)) \in P_{1}(\mathcal{T})$ equals $(x-\operatorname{mid}(T))$ at $x \in T \in \mathcal{T}$. Hence,

$$
\begin{aligned}
\left\|\nabla u-\nabla_{\mathrm{NC}} u_{\mathrm{CR}}\right\| & \leq\left\|\nabla u-p_{\mathrm{RT}}\right\|+\left\|p_{\mathrm{RT}}-\nabla_{\mathrm{NC}} \tilde{u}_{\mathrm{CR}}\right\|+\left\|\nabla_{\mathrm{NC}} \bar{u}_{\mathrm{CR}}-\nabla_{\mathrm{NC}} u_{\mathrm{CR}}\right\| \\
& \lesssim\left\|\nabla u-p_{\mathrm{RT}}\right\|+\|h f\| .
\end{aligned}
$$

This proves the first inequality in Theorem 2.3. The proof of the second one exploits Marini's identity again:

$$
\left\|\nabla u-p_{\mathrm{RT}}\right\| \leq\left\|\nabla u-\nabla_{\mathrm{NC}} \bar{u}_{\mathrm{CR}}\right\|+\left\|\nabla_{\mathrm{NC}} \bar{u}_{\mathrm{CR}}-p_{\mathrm{RT}}\right\| \lesssim\left\|\nabla u-\nabla_{\mathrm{NC}} u_{\mathrm{CR}}\right\|+\|h f\| .
$$

The efficiency of $\|h f\|$ up to oscillations [32], namely,

$$
\|h f\| \lesssim\left\|\nabla u-\nabla_{\mathrm{NC}} u_{\mathrm{CR}}\right\|+\operatorname{osc}(f, \mathcal{T})
$$

concludes the proof.

This statement is also included in [7].

3.4. Proof of Theorem 2.4. It appears instructive to start a general indirect proof to point out where the compactness comes into the play which is then followed by a perturbation argument.

Step 1. Let $\operatorname{PMP}(\cdot), \operatorname{CFEM}(\cdot)$, and $\operatorname{RTMFEM}(\cdot)$ denote the solution operators associated with (1.1), (2.1.b), and (2.3.b). If the theorem is false, there is a sequence of right-hand sides $f_{n} \in L^{2}(\Omega)$ with corresponding solutions $u_{n}:=\operatorname{PMP}\left(f_{n}\right)$ and approximations $u_{\mathrm{C}}(n):=\operatorname{CFEM}\left(f_{n}\right)$ and $p_{\mathrm{RT}}(n):=\operatorname{RTMFEM}\left(f_{n}\right)$ such that

$$
u\left(\left\|\nabla u_{n}-p_{\mathrm{RT}}(n)\right\|+\operatorname{osc}\left(f_{n}, \mathcal{T}\right)\right) \leq\left\|\nabla\left(u_{n}-u_{\mathrm{C}}(n)\right)\right\| .
$$

Step 2. Since the aforementioned operators $\operatorname{PMP}(\cdot), \operatorname{CFEM}(\cdot), \operatorname{RTMFEM}(\cdot)$ are homogeneous of degree one, the scaling $f_{n} /\left\|f_{n}\right\|$ in (3.12) leads to a new sequence of right-hand sides $f_{n}$ of $L^{2}$ norm one which satisfies (3.12). In other words, we may and will assume without loss of generality that $f_{n}$ in (3.12) satisfies

$$
\left\|f_{n}\right\|=1 \text { for all } n \in \mathbb{N} \text {. }
$$

Step 3. Since the right-hand sides $f_{n}$ are bounded in (3.13), there exists a subsequence $n_{j}$ with $f_{n_{j}} \rightarrow f_{\infty}$ in $L^{2}(\Omega)$. Rellich's compactness embedding theorem leads to strong convergence $f_{n} \rightarrow f_{\infty}$ in $H^{-1}(\Omega)$. Since $\operatorname{PMP}(\cdot): H^{-1}(\Omega) \rightarrow H_{0}^{1}(\Omega)$ is a Riesz isomorphism, it follows that $u_{n,} \rightarrow u_{\infty}$ in $H_{0}^{1}(\Omega)$. The stability of the discrete approximation operators also imply boundedness in the discrete spaces (recall that $\mathcal{T}$ is fixed). For a selection of a further subsequence (not relabeled), strong convergence follows. In other words, we may and will assume without loss of generality that the subsequence $n_{j}$ leads to

$$
\begin{array}{rr}
f_{n_{j}} \rightarrow f_{\infty} \text { in } H^{-1}(\Omega), & u_{n_{j}} \rightarrow u_{\infty} \text { in } H_{0}^{1}(\Omega), \\
p_{\mathrm{RT}}\left(n_{j}\right) \rightarrow p_{\mathrm{RT}}(\infty) \text { in } \mathrm{RT}_{0}(\mathcal{T}), & u_{\mathrm{C}}\left(n_{j}\right) \rightarrow u_{\mathrm{C}} \text { in } V_{\mathrm{C}}(\mathcal{T}) .
\end{array}
$$


Step 4. Since the right-hand side in (3.12) is bounded as $j \rightarrow \infty$ and the left-hand side involves $n_{j} \rightarrow \infty$,

$$
\lim _{j \rightarrow \infty}\left(\left\|\nabla u_{n},-p_{\mathrm{RT}}\left(n_{j}\right)\right\|+\operatorname{osc}\left(f_{n_{j}}, \mathcal{T}\right)\right)=0 .
$$

This and the convergence (3.14) imply that

$$
p_{\mathrm{RT}}(\infty)=\nabla u_{\infty} \quad \text { and } \quad \operatorname{osc}\left(f_{\infty}, \mathcal{T}\right)=0
$$

In other words, the solution $u_{\infty}=\operatorname{PMP}\left(f_{\infty}\right)$ for $f_{\infty} \in P_{0}(\mathcal{T})$ satisfies $\nabla u_{\infty} \in \operatorname{RT}_{0}(\mathcal{T})$.

Step 5. Elliptic regularity for the Poisson model problem with homogeneous Dirichlet boundary conditions along the entire boundary states that $u_{\infty} \in H_{0}^{1}(\Omega) \cap$ $H^{1+s}(\Omega)$ for $s>1 / 2$. Hence, $\nabla u_{\infty} \in \mathrm{RT}_{0}(\mathcal{T})$ is continuous. Some elementary calculations on two Raviart-Thomas functions which are globally continuous on an edge patch $\omega_{E}$ of an interior edge $E$ show that they need to coincide in the sense that $\nabla u_{\infty}$ is affine on $\omega_{E}$. Since the interior edge patches are overlapping, this shows that $u_{\infty}$ is a quadratic polynomial on $\Omega$ with homogeneous boundary data. Some typical example on a circle is provided in the counterexample below. On a polygonal domain, the fact that there is some corner specifies the quadratic polynomial $u_{\infty}$ to be some particular edge-bubble function. This, in turn, shows that $u_{\infty} \equiv 0$ is the only remaining solution. This implies $f_{\infty} \equiv 0$.

Step 6. The point is that $f_{\infty} \equiv 0$ is not at all a contradiction to (3.13)-(3.14) for general right-hand sides in $L^{2}(\Omega)$. Thus we restrict the above arguments to the class of right-hand sides $f$ and $f_{n}$ in $P_{0}(\mathcal{T})$. The finite dimension then yields strong convergence in $P_{0}(\mathcal{T})$ endowed with any norm, e.g., endowed with the $L^{2}(\Omega)$ norm. This leads to the contradiction $f_{n} \rightarrow f_{\infty}$ and $\left\|f_{n},\right\|=1$ implies $\left\|f_{\infty}\right\|=1$. In other words, steps 1-6 lead to an indirect proof of the following proposition of step 7.

Step 7. Proposition: Given any regular triangulation $\mathcal{T}$ of the polygonal Lipschitz domain $\Omega$ in $\mathbb{R}^{2}$ into triangles, there exists some constant $\bar{C}(\mathcal{T})$ such that for any right-hand side $\tilde{f} \in P_{0}(\mathcal{T})$ and $\tilde{u}=\operatorname{PMP}(\bar{f})$ the associated conforming $P_{1}$ FEM solution $\tilde{u}_{\mathrm{C}}=\operatorname{CFEM}(\tilde{f})$ and the RT-MFEM solution $\tilde{p}_{\mathrm{RT}}=\operatorname{RTMFEM}(\tilde{f})$ satisfy

$$
\left\|\nabla\left(\tilde{u}-\bar{u}_{\mathrm{C}}\right)\right\| \leq \tilde{C}(\mathcal{T})\left\|\nabla \tilde{u}-\tilde{p}_{\mathrm{R} T}\right\| .
$$

The proof is with steps $1-6$ and the observation that piecewise constant functions have no oscillations.

Step 8. The indicated perturbation concerns an arbitrary right-hand side $f \in$ $L^{2}(\Omega)$ and its piecewise integral mean $\tilde{f}:=\Pi_{0} f \in P_{0}(\mathcal{T})$. The original solution $u=\operatorname{PMP}(f)$ compares to $\tilde{u}=\operatorname{PMP}(\bar{f})$ via

$$
\|\nabla(u-\tilde{u})\| \leq \operatorname{osc}(f, \mathcal{T}) / \pi .
$$

The proof is a standard exercise with the weak form or $e:=u-\tilde{u}$ and

$$
\|\nabla e\|^{2}=\int_{\Omega}\left(f-\Pi_{0} f\right) e \mathrm{~d} x=\int_{\Omega}\left(f-\Pi_{0} f\right)\left(e-\Pi_{0} e\right) \mathrm{d} x
$$

followed with a weighted Cauchy inequality with the mesh-size $h_{\mathcal{T}} \in P_{0}(\mathcal{T})$ and the piecewise Poincare inequality with Payne-Weinberger constant for the convex triangles. The same proof also verifies the discrete analogue

$$
\left\|\nabla\left(u_{C}-\tilde{u}_{C}\right)\right\| \leq \operatorname{osc}(f, \mathcal{T}) / \pi .
$$

Notice that $p_{\mathrm{RT}}=\operatorname{RTMFEM}(f)=\operatorname{RTMFEM}(\tilde{f})=\tilde{p}_{\mathrm{RT}}$. 
Step 9. The combination of (3.15)-(3.17) show with appropriate triangle inequalities that

$$
\begin{aligned}
\left\|\nabla\left(u-u_{\mathrm{C}}\right)\right\| & \lesssim\left\|\nabla\left(\tilde{u}-\tilde{u}_{\mathrm{C}}\right)\right\|+\operatorname{osc}(f, \mathcal{T}) \\
& \leq \tilde{C}(\mathcal{T})\left(\left\|\nabla \tilde{u}-\tilde{p}_{\mathrm{RT}}\right\|+\operatorname{osc}(f, \mathcal{T})\right) \lesssim \tilde{C}(\mathcal{T})\left(\left\|\nabla u-p_{\mathrm{RT}}\right\|+\operatorname{osc}(f, \mathcal{T})\right)
\end{aligned}
$$

This concludes the proof with a constant $C(\mathcal{T})$ which depends on $\bar{C}(\mathcal{T})$ and, hence, on the interior angles in $\mathcal{T}$.

3.5. Proof of Theorem 2.5. The counterexample concerns the quadratic polynomial $u_{B}$,

$$
u_{B}(x):=\left(1-|x|^{2}\right) / 2 \quad \text { in the unit disk } B=B(0,1) .
$$

Note that $u_{B} \in C^{\infty}\left(\mathbb{R}^{2}\right)$ solves $-\Delta u=2$ in $B$ and $u=0$ on $\partial B$. Moreover, $\nabla u=$ $-x \in \operatorname{RT}_{0}(\mathcal{T})$.

Given a small $0<h \ll 1$ as the uniform edge-length of a regular polygon $\Omega$ with vertices on $\partial B$, let $\mathcal{T}$ denote a shape-regular quasi-uniform triangulation of $\Omega$ with maximal mesh-size $\approx h$. Let $u \in H_{0}^{1}(\Omega)$ solve $-\Delta u=2$ in $\Omega$.

The point of departure is the claim $\left\|\nabla\left(u-u_{B}\right)\right\| \lesssim h^{3 / 2}$. To prove this, observe that, since $u_{B}-u$ is harmonic with boundary values $\left.u_{B}\right|_{\partial \Omega}$,

$$
\left\|\nabla\left(u-u_{B}\right)\right\|=\min \left\{\|\nabla v\|\left|v \in H^{1}(\Omega): v\right|_{\partial \Omega}=\left.u_{B}\right|_{\partial \Omega}\right\} .
$$

Therefore it remains to design some function $w \in H^{1}(\Omega)$ with $\left.w\right|_{\partial \Omega}=\left.u_{B}\right|_{\partial \Omega}$ and $\|\nabla w\| \lesssim h^{3 / 2}$. To do so, set, for any $E \in \mathcal{E}$ with $E=\operatorname{conv}\{a, b\}=\partial \Omega \cap T$ for some $T \in \mathcal{T}$ and nodal basis functions $\varphi_{a}$ and $\varphi_{b}$ of the Courant FEM,

$w_{E}:=\frac{1}{2} h^{2} \varphi_{a} \varphi_{b} \in H^{1}(\Omega)$ with $\operatorname{supp} w_{E}=T$ and $w=\left(\sum_{E \in \mathcal{E}: E \subset \partial \Omega} w_{E}\right) \in H^{1}(\Omega)$.

Since $w_{E}(x)=u_{B}(x)$ for all $x \in E$, and $\left\|\nabla w_{E}\right\| \approx h^{2}$, it follows that

$$
\left\|\nabla\left(u-u_{B}\right)\right\| \leq \sqrt{\sum_{E \in \mathcal{E}: E \subset \partial \Omega}\left\|\nabla w_{E}\right\|^{2}} \approx h^{3 / 2} .
$$

For $Q(f, \mathcal{T}):=\left\{q_{\mathrm{RT}} \in \mathrm{RT}_{0}(\mathcal{T}) \mid \operatorname{div} q_{\mathrm{RT}}=-\Pi_{0} f\right\}$, the RT-MFEM approximation $p_{\mathrm{RT}}$ of $\nabla u$ on $\Omega$ is characterized by

$$
\left\|\nabla u-p_{\mathrm{RT}}\right\|=\min \left\{\left\|\nabla u-q_{\mathrm{RT}}\right\| \mid q_{\mathrm{RT}} \in Q(2, \mathcal{T})\right\} .
$$

This is well understood in the context of minimization under side restrictions and its connection to saddle-point problems [6]. Since $\nabla u_{B} \in Q(2, \mathcal{T})$, it follows that

$$
\left\|\nabla u-p_{\mathrm{RT}}\right\| \leq\left\|\nabla\left(u-u_{B}\right)\right\| \lesssim h^{3 / 2} .
$$

Since $f \equiv 2$, it holds that osc $f, \mathcal{T}=0$ and $\|h f\| \approx h$. Hence, (3.11) and (3.18) imply

$$
C h^{-1 / 2}\left(\left\|\nabla u-p_{\mathrm{RT}}\right\|+\operatorname{osc}(f, \mathcal{T})\right) \leq\left\|\nabla u-\nabla_{\mathrm{NC}} u_{\mathrm{CR}}\right\| .
$$

Given $M>0$, the choice $h=(C / M)^{2}$ proves the assertion of Theorem 2.5. 


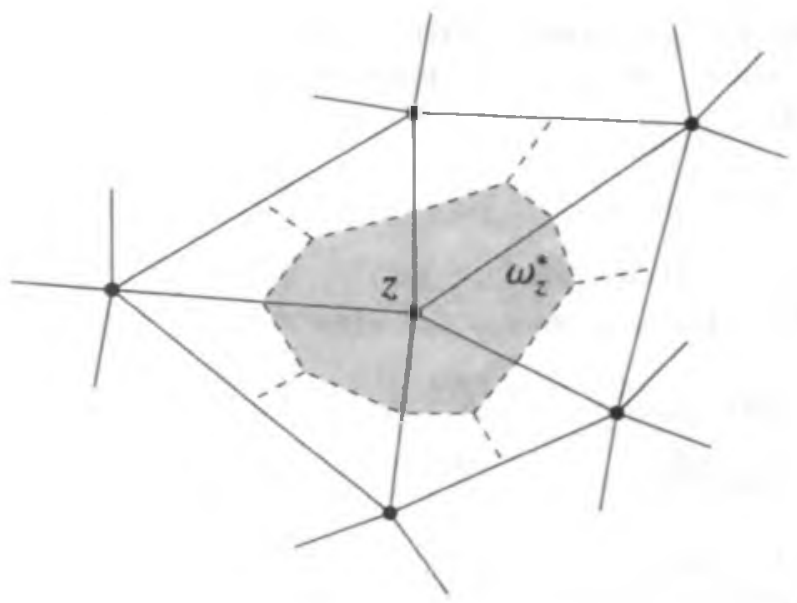

FIG. 4.1. The control volume $\omega_{z}^{*}$.

4.3.2. DGFEM on nonconforming meshes. Often, the large number of degrees of freedom in DGFEM compared to CFEM is justified by the possibility of using nonconforming meshes. These meshes may contain some finite number of hanging nodes per edge. Define $V_{\mathrm{DG}}^{k}(\mathcal{T}):=P_{k}(\mathcal{T})$ for some nonconforming triangular mesh $\mathcal{T}$. It is shown in [26] that also for such meshes there exists an averaging operator $\mathrm{I}_{\mathrm{C}}: V_{\mathrm{DG}}^{k}(\mathcal{T}) \rightarrow V$ that satisfies (2.8) with suitably redefined jump seminorm. The image $\mathrm{I}_{\mathrm{C}}\left(V_{\mathrm{DG}}^{k}(\mathcal{T})\right)=V_{\mathrm{DG}}^{k}(\mathcal{T}) \cap V$ defines some conforming space $V_{\mathrm{C}}^{k}(\mathcal{T})$. One might not want to use $V_{\mathrm{C}}^{k}(\mathcal{T})$ for actual computations but the corresponding Galerkin solution $u_{\mathrm{C}}^{k}$ serves for a comparison. The proof of Theorem 2.2 in section 3.2 remains valid in this setting and establishes the comparison

$$
\left\|\nabla u-\nabla u_{\mathrm{C}}^{k}\right\| \lesssim\left\|u-u_{\mathrm{DG}}^{k}\right\|_{\mathrm{DG}} \lesssim\left\|\nabla u-\nabla u_{\mathrm{C}}^{k}\right\|+\operatorname{osc}_{k}(f, \mathcal{T})
$$

for nonconforming meshes. Hence, even on nonconforming meshes, the accuracy of DGFEM is limited by the accuracy that is provided by its largest conforming subspace. Analogous results hold for nontriangular meshes.

4.4. Results for finite volume FEM. A common way [23] to design the control volumes $\omega_{z}^{*}$ for each node $z \in \mathcal{N}(\Omega)$ for the finite volume finite element method (FVFEM or vertex-based finite volumes) [18] is to connect the barycenter of the triangles of $\mathcal{T}$ with the midpoints of their edges as depicted in Figure 4.1. Then FVFEM seeks $u_{\mathrm{FV}} \in V_{\mathrm{C}}(\mathcal{T})$ with

$$
\left(\int_{\Omega} \nabla u_{\mathrm{FV}} \cdot \nabla \varphi_{z} \mathrm{~d} x=\right) \int_{\partial \omega_{*}^{*}} \nabla u_{\mathrm{FV}} \cdot \nu \mathrm{d} s=\int_{\omega_{*}^{*}} f \mathrm{~d} x \quad \text { for all } z \in \mathcal{N}(\Omega),
$$

where $\varphi_{z} \in V_{\mathrm{C}}(\mathcal{T})$ is the nodal basis function with $\varphi_{z}(z)=1$ and $\varphi_{z}(y)=0$ for all $y \in \mathcal{N} \backslash\{z\}$. Hence, CFEM and FVFEM differ only by the right-hand sides [18].

Define $e(z):=\left(u_{\mathrm{C}}(z)-u_{\mathrm{FV}}(z)\right) \in \mathbb{R}, z \in \mathcal{N}$, through

$$
u_{\mathrm{C}}-u_{\mathrm{FV}}=\sum_{z \in \mathcal{N}} e(z) \varphi_{z}
$$

and the characteristic function $\left.\chi_{z}^{*}\right|_{\omega_{z}^{*}} \equiv 1$ and $\left.\chi_{z}^{*}\right|_{\Omega \backslash \omega_{z}^{*}} \equiv 0$ and let $e_{T} \in \mathbb{R}$ for any 
$T \in \mathcal{T}$. The fact that $f_{T}\left(\varphi_{z}-\chi_{z}^{*}\right) \mathrm{d} x=0$ for all $T \in \mathcal{T}$ leads to

$$
\begin{aligned}
& \left\|\nabla\left(u_{\mathrm{C}}-u_{\mathrm{FV}}\right)\right\|^{2}=\int_{\Omega}\left(f-\Pi_{0} f\right)\left(u_{\mathrm{C}}-u_{\mathrm{FV}}-\sum_{z \in \mathcal{N}} e(z) \chi_{z}^{*}\right) \mathrm{d} x \\
& \leq \operatorname{osc}(f, \mathcal{T})\left(\left\|\nabla\left(u_{\mathrm{C}}-u_{\mathrm{FV}}\right)\right\|+\sum_{T \in \mathcal{T}} \operatorname{diam}(T)^{-1}\left\|\sum_{z \in \mathcal{N}(T)} e(z) \chi_{z}^{*}-e_{T}\right\|_{L^{2}(T)}\right) .
\end{aligned}
$$

Since the eigenvalues of the mass matrix $M:=\left(\int_{T} \varphi_{z_{j}} \varphi_{z_{k}} \mathrm{~d} x\right)_{j, k=1,2,3}$ are larger than $|T| / 12$, any $T \in \mathcal{T}$ with vertices $z_{1}, z_{2}, z_{3}$ satisfies

$$
\begin{aligned}
\left\|\sum_{z \in \mathcal{N}(T)} e(z) \chi_{z}^{*}-e_{T}\right\|_{L^{2}(T)}^{2}=|T| / 3 \sum_{z \in \mathcal{N}(T)}\left(e(z)-e_{T}\right)^{2} \\
\leq 4\left(\begin{array}{c}
e\left(z_{1}\right)-e_{T} \\
e\left(z_{2}\right)-e_{T} \\
e\left(z_{3}\right)-e_{T}
\end{array}\right) \quad M\left(\begin{array}{l}
e\left(z_{1}\right)-e_{T} \\
e\left(z_{2}\right)-e_{T} \\
e\left(z_{3}\right)-e_{T}
\end{array}\right) \\
\leq 4\left\|\sum_{z \in \mathcal{N}(T)}\left(e(z)-e_{T}\right) \varphi_{z}\right\|_{L^{2}(T)}^{2}=4\left\|u_{C}-u_{F V}-e_{T}\right\|_{L^{2}(T)}^{2} .
\end{aligned}
$$

The choice $e_{T}:=f_{T} u_{\mathrm{C}}-u_{\mathrm{FV}} \mathrm{d} x$ and a piecewise Poincaré inequality then yield

$$
\left\|\nabla\left(u_{\mathrm{C}}-u_{\mathrm{FV}}\right)\right\| \lesssim \operatorname{osc}(f, \mathcal{T}) .
$$

Hence, $\left\|\nabla\left(u-u_{\mathrm{C}}\right)\right\|+\operatorname{osc}(f, \mathcal{T}) \approx\left\|\nabla\left(u-u_{\mathrm{FV}}\right)\right\|+\operatorname{osc}(f, \mathcal{T})$.

5. Numerical illustration. The first experiment illustrates the counterexample of Theorem 2.5 which appears to be nongeneric. The second example with a cornersingularity shows equality of convergence rates as a typical behavior.

5.1. Illustration of Theorem 2.5. In the first experiment, the domains $\Omega_{j}$, $j=2, \ldots, 9$, are regular polygons with $2^{j}$ edges whose vertices lie on the unit sphere $\partial B(0,1)$ as in the proof of Theorem 2.5. For each domain $\Omega_{j}$ a series of red-refined triangulations $\mathcal{T}_{, \ell}:=\operatorname{red}^{(\ell)}\left(\mathcal{T}_{j}\right)$ of an initial triangulation $\mathcal{T}_{j}$ determines the discrete solutions $u_{\mathrm{CR}}^{(j, \ell)}$ and $p_{\mathrm{RT}}^{(j, \ell)}$ of $(2.2 . \mathrm{b})$ and (2.3.b). For the red-refinement of the triangulation each triangle is refined as in Figure 2.1. The initial triangulations are given as follows. $\mathcal{T}_{2}$ is the criss-cross triangulation and given the triangulation $\mathcal{T}_{\text {, a }}$ red-refinement $\operatorname{red}\left(\mathcal{T}_{j}\right)$ of $\mathcal{T}_{j}$ is modified in that the new boundary nodes are projected to the circle $\partial B(0,1)$. This defines $\mathcal{T}_{j+1}$; the triangulations $\mathcal{T}_{2}, \mathcal{T}_{3}, \mathcal{T}_{0}$ are depicted in Figure 5.1.

Table 5.1 contains the quotients of the flux errors with exact solution $u^{(j)}$ based on the Poisson model problem on $\Omega_{j}$ and its flux approximations $\nabla_{\mathrm{NC}} u_{\mathrm{CR}}^{(j, \ell)}$ and $p_{\mathrm{RT}}^{(j, \ell)}$ based on the triangulation $\mathcal{T}_{, \ell}$,

$$
q(\ell, j):=\frac{\left\|\nabla u^{(j)}-p_{\mathrm{RT}}^{(j, \ell)}\right\|}{\left\|\nabla u^{(j)}-\nabla_{\mathrm{NC}} u_{\mathrm{CR}}^{(j, \ell)}\right\|} .
$$

The convergence history plot of Figure 5.2 shows the flux errors plotted against the number of degrees of freedom. The crosses and the triangles mark the errors for the 

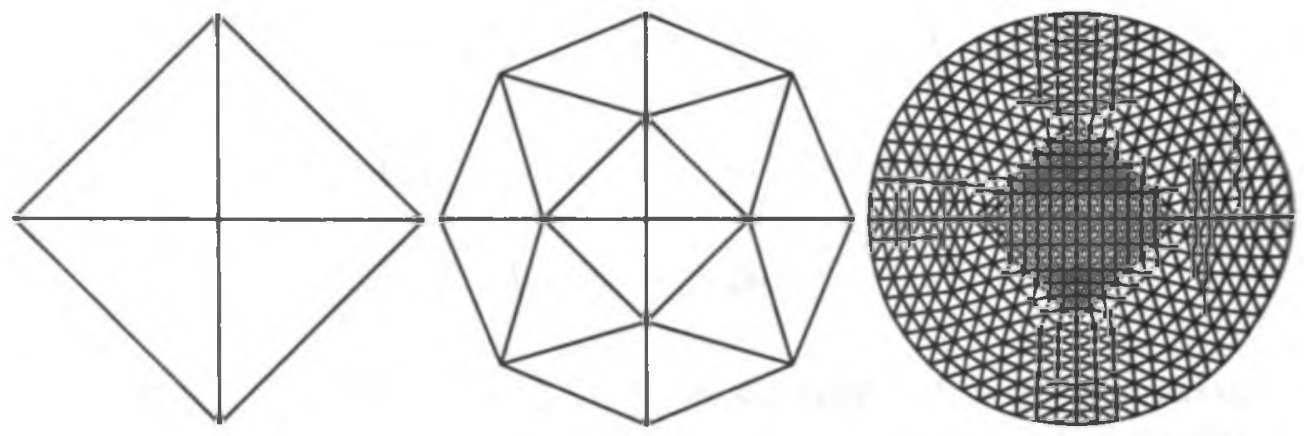

FIG. 5.1. $\mathcal{T}_{2}, \mathcal{T}_{3}$, and $\mathcal{T}_{6}$ from subsection 5.1 .

TABLE 5.1

Quotient $q(\ell, j)$ from (5.1) for RT-MFEM and CR-NCFEM.

\begin{tabular}{c|cccccccc}
\hline & $\ell=0$ & 1 & 2 & 3 & 4 & 5 & 6 & 7 \\
\hline$j=2$ & .94097 & .90727 & .90405 & .90346 & .90335 & .90333 & .90333 & .90333 \\
3 & .51218 & .72172 & .77759 & .80313 & .81703 & .82517 & .83011 & \\
4 & .34413 & .56908 & .66257 & .71381 & .74579 & .76746 & & \\
5 & .23909 & .43216 & .53576 & .60128 & .64662 & & & \\
6 & .16793 & .31867 & .41264 & .47906 & & & & \\
7 & .11856 & .23084 & .30735 & & & & & \\
8 & .08424 & .16622 & & & & & & \\
9 & .06103 & & & & & & & \\
\hline
\end{tabular}

Crouzeix-Raviart and the Raviart-Thomas solution. In order to compute the error, for each domain $\Omega_{j}$ some $P_{2}$ reference solution is computed on $\mathcal{T}_{j, 11-j}$. The dashed lines connect the errors for the triangulations $\mathcal{T}_{2}, \mathcal{T}_{3}, \ldots \mathcal{T}_{9}$ of the proof of Theorem 2.5 and show the expected convergence rates.

The Raviart-Thomas errors show a larger convergence rate on the initial triangulations than the Crouzeix-Raviart errors, while for a fixed domain the RaviartThomas errors converge with the same convergence rate as the Crouzeix-Raviart errors after a very long preasymptotic plateaux. The same behavior can be observed in Table 5.1: For a fixed $\ell$ the quotients $q(\ell, j)$ are decreasing while for a fixed $j$ the quotients first increase and then stay on the same level.

Since the errors of CR-NCFEM and CFEM are equivalent, the reciprocal of the quotients $q(\ell, j)$ may serve as a lower bound for the constant $C\left(\mathcal{T}_{, \ell}\right)$ in Theorem 2.4 (up to some multiplicative constant which does not depend on the domain or the mesh-size). This lower bound $q(\ell, j)^{-1}$ increases with $j$ in this experiment. However, Table 5.1 strongly suggests that $q(\ell, j)^{-1}$ remains bounded as $\ell$ increases. These experimental results confirm the conjecture from Remark 3 which says that the constant $C(\mathcal{T})$ from Theorem 2.4 depends only on the domain $\Omega$ and interior angles of $\mathcal{T}$ but not on the mesh-size.

5.2. Numerical comparison on L-shaped domain. The second example is devoted to a prototypical equivalent behavior of Courant FEM, CR-NCFEM, DGFEM, and RT-MFEM. The corner singular functions on a typical corner of a polygonal domain $\Omega \subset \mathbb{R}^{2}$, for instance, do not allow for the improved convergence of RT-MFEM. 


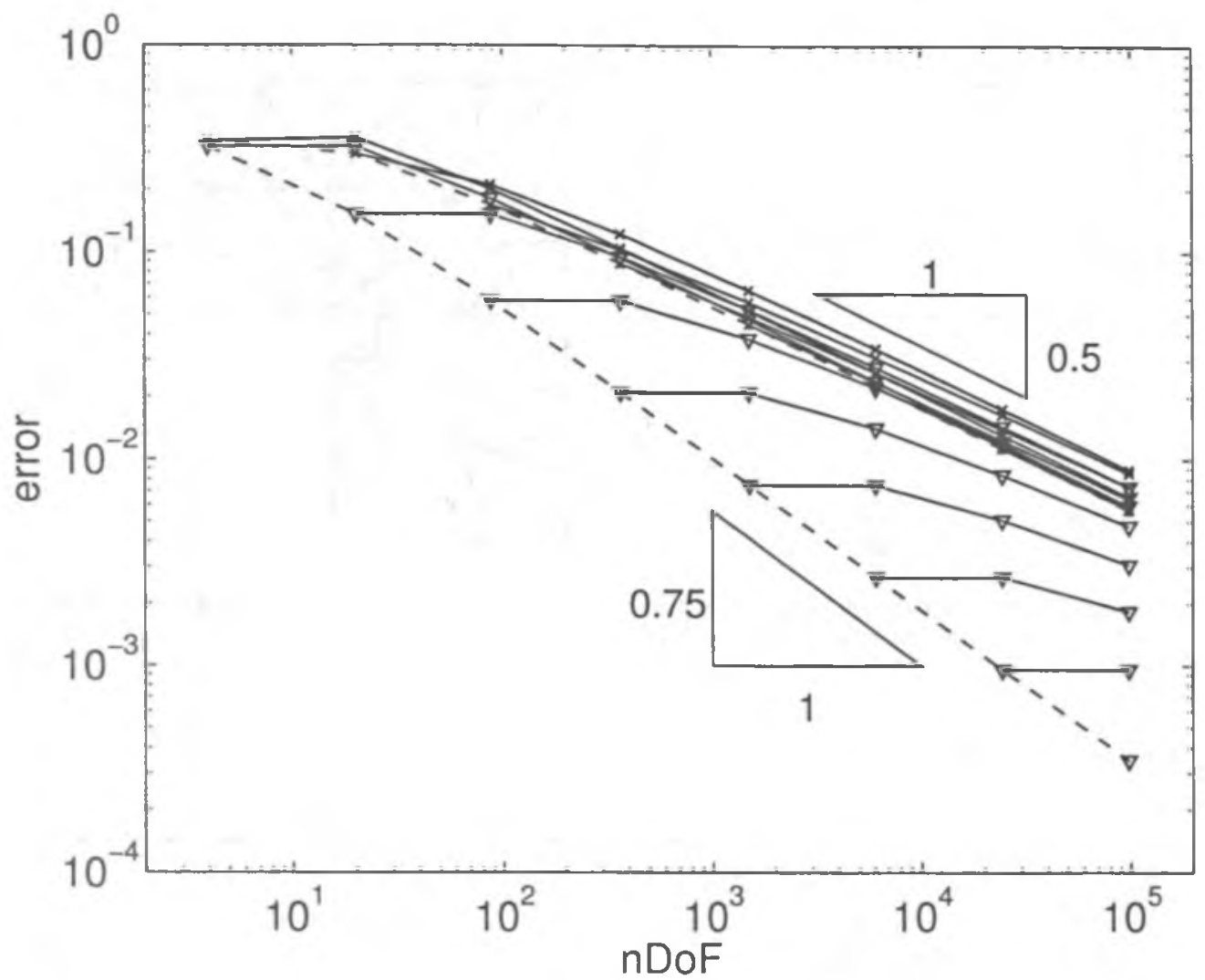

Fig. 5.2. Errors of $C R-N C F E M(\mathrm{x})$ and $R T-M F E M(\nabla)$ in subsection 5.1.

To illustrate this, let the origin 0 be a nonconvex vertex of $\partial \Omega$ with maximal interior angle $\omega$ such that, up to some smooth truncation function, the leading singular function has the form

$$
u_{s i n g}(r, \varphi)=r^{\alpha} \sin (\alpha \varphi) \text { for } 0<r<1 \text { and } 0<\varphi<\omega
$$

with $1 / 2<\alpha:=\pi / \omega<1$. Given any triangle $T$ with vertex 0 , the approximation error of the flux $\nabla u_{s i n g}=a r^{\alpha-1}(\sin \alpha \varphi, \cos \alpha \varphi)$ by Raviart-Thomas functions is bounded from below by

$$
\min _{a, b, c \in \mathbb{R}}\left\|\nabla u_{s i n g}(x)-(a, b)-c x\right\|_{L^{2}(T)} \approx h_{T}^{\alpha}
$$

and, hence, is of the same order as the interpolation error of the piecewise affine nodal or edgewise interpolation in $V_{C}(\mathcal{T})$ or $\mathrm{CR}_{0}^{1}(\mathcal{T})$. For meshes where this defines the convergence rates like in the numerical examples below, this shows that RT-MFEM has the same order of convergence and is not superior to the remaining finite element schemes.

The L-shaped domain $\Omega=[-1,1]^{2} \backslash([0,1] \times[-1,0])$ illustrates this with the righthand side $f \equiv 2$. The discontinuous Galerkin schemes under consideration are the symmetric interior penalty method and the nonsymmetric interior penalty method 

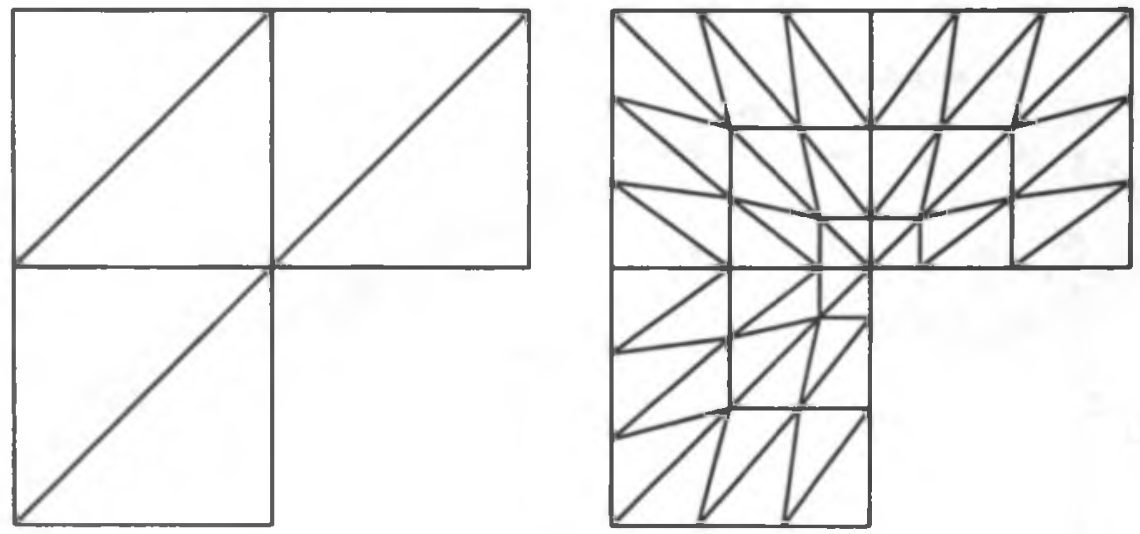

FIG. 5.3. The initial triangulation for the red-refined triangulations (left) and a graded mesh (right) of the L-shaped domain from subsection 5.2 .

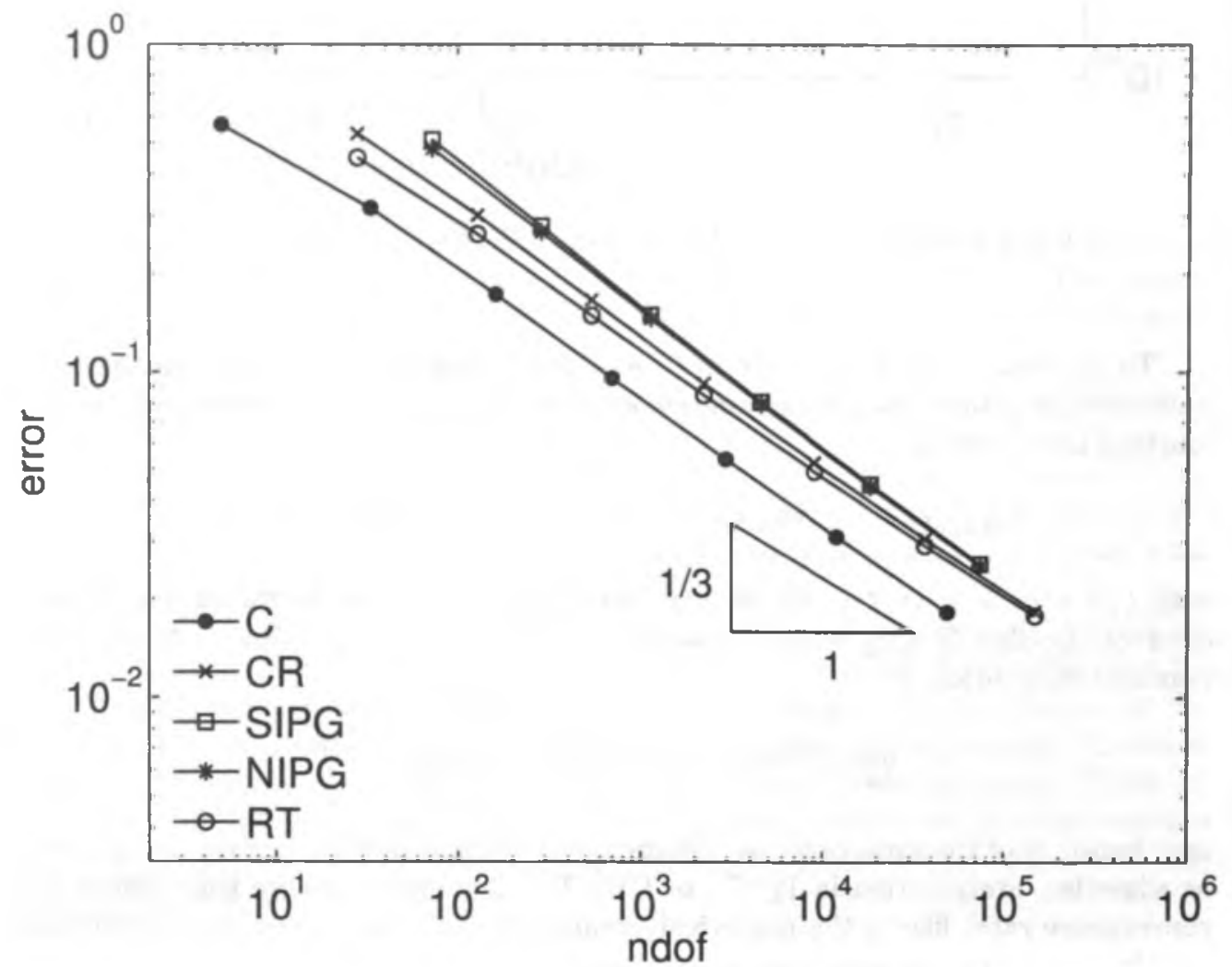

FIG. 5.4. Errors of CFEM, CR-NCFEM, SIPG, NIPG, and RT-MFEM for uniform red-refined meshes on the L-shaped domain. 


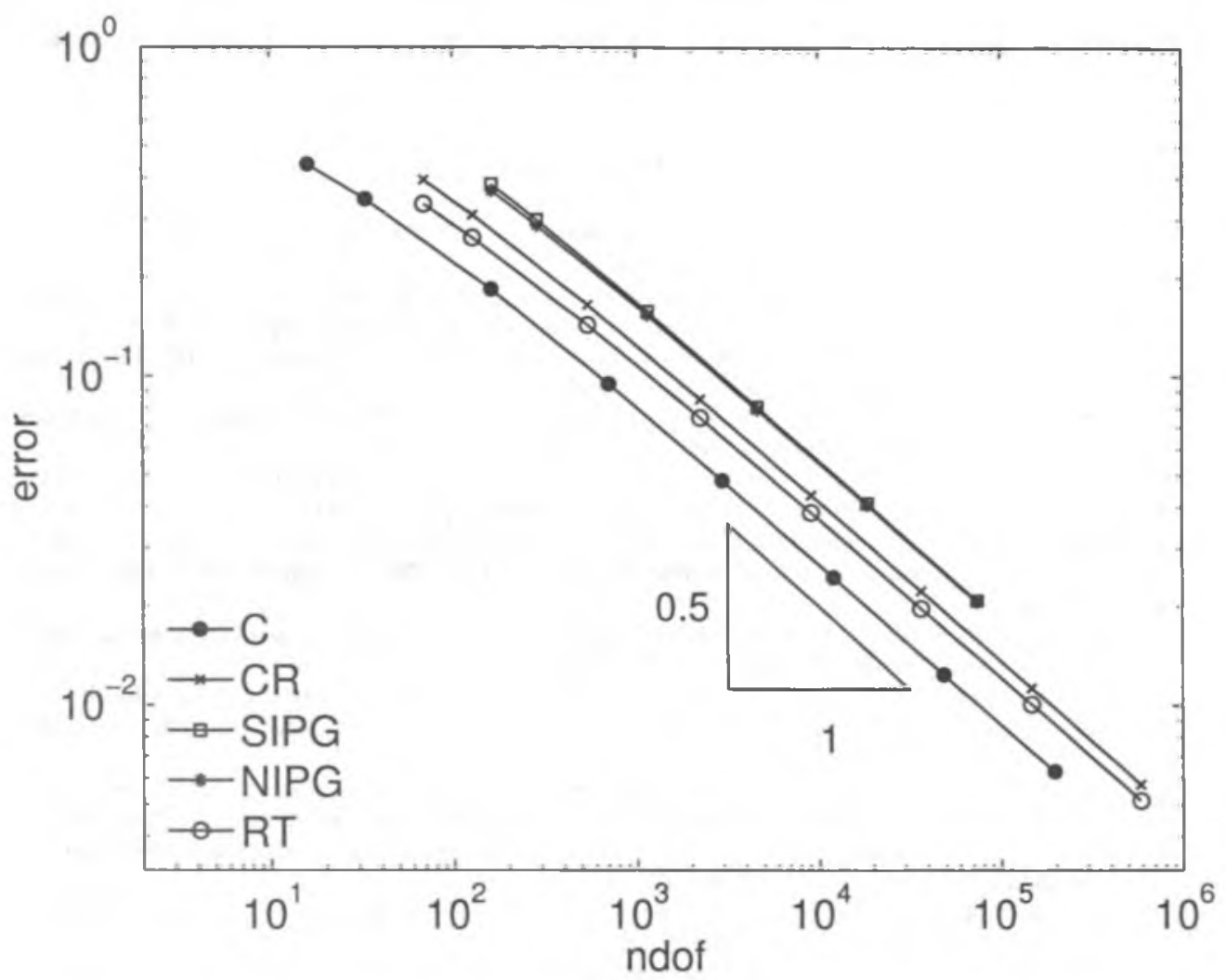

FIG. 5.5. Errors of CFEM, CR-NCFEM, SIPG, NIPG, and RT-MFEM for graded meshes on the $L$-shaped domain.

with the bilinear forms

$$
\begin{aligned}
a_{\mathrm{DG}}\left(u_{\mathrm{DG}}, v_{\mathrm{DG}}\right):=\int_{\Omega} \nabla_{\mathrm{NC}} u_{\mathrm{DG}} \cdot \nabla_{\mathrm{NC}} v_{\mathrm{DG}} \mathrm{d} x-\delta \sum_{E \in \mathcal{E}} \int_{E}\left[u_{\mathrm{DG}}\right]_{E}\left\langle\nabla_{\mathrm{NC}} v_{\mathrm{DG}}\right\rangle_{E} \cdot \nu_{E} \mathrm{~d} s \\
-\sum_{E \in \mathcal{E}} \int_{E}\left[v_{\mathrm{DG}}\right]_{E}\left\langle\nabla_{\mathrm{NC}} u_{\mathrm{DG}}\right\rangle_{E} \cdot \nu_{E} \mathrm{~d} s+\sum_{E \in \mathcal{E}} \frac{\eta}{|E|} \int_{E}\left[u_{\mathrm{DG}}\right]_{E}\left[v_{\mathrm{DG}}\right]_{E} \mathrm{~d} s
\end{aligned}
$$

for $\delta=1$ for SIPG and $\delta=-1$ for NIPG and penalty parameter $\eta=10$.

The solutions of (2.1.b), (2.2.b), (2.7), and (2.3.b) are computed on a sequence of red-refined triangulations $\tau_{0}, \mathcal{T}_{1}, \ldots, \mathcal{T}_{0}$ and a sequence of graded meshes $\mathcal{T}_{j}{ }^{G}$ for $j=3,4,8,16,32,64,128,256$ with grading parameter $\beta=3 / 2$, where $j$ denotes the vertices on one side of one macro triangle. The initial triangulation $\overline{0}$ for the redrefined triangulations and the graded mesh $\mathcal{T}_{3}{ }^{G}$ are depicted in Figure 5.3. The errors for solutions on a red-refined triangulation are computed by a $P_{2}$ reference solution on $\mathcal{T}_{9}$ and the errors for a solution on a graded mesh $\mathcal{T}_{j}^{G}$ are computed by a $P_{2}$ reference solution on $\operatorname{red}^{(2)}\left(\mathcal{T}_{j}{ }^{G}\right)$. Figures 5.4 and 5.5 reveal the expected convergence rates $1 / 3$ (resp., 1/2) for uniform (resp., graded) meshes for all three methods. The equivalence of the three methods is clearly visible.

Note added in proof. After this work was completed, the authors learned that the statements of Lemma 5.1 and Proposition 5.2 in [5] result in equivalence of conforming FEM with SIPG for very large penalization with an independent proof. Hence the 
result of Theorem 2.2 of this paper generalizes that partial result to a larger class of DG schemes.

\section{REFERENCES}

[1] D. N. ARNOLD, An interior penalty finite element method with discontinuous elements, SIAM J. Numer. Anal., 19 (1982), pp. 742-760.

[2] R. BECKER. S. MAO. AND Z. SHI, A convergent nonconforming adaptive finite element method with quasi-optimal complexity, SIAM J. Numer. Anal., 47 (2010). pp. 4639-4659.

[3] P. Binev: W. DAhmen. AND R. DEVore. Adaptive finite element methods with convergence rates. Numer. Math.. 97 (2004), pp. 219-268.

[4] P. B. Bochev And M. D. Gunzburger. Least-squares Finite Element Methods, Appl. Math. Sci.. 166, Springer, New York, 2009.

[5] A. BONITO AND R. H. Nochetro, Quasi-optimal convergence rate of an adaptive discontinuous Galerkin method, SIAM J. Numer. Anal., 48 (2010), pp. 734-771.

[6] D. Braess, Finite Elements, 3rd ed., Theory, Fast Solvers, and Applications in Elasticity Theory, Cambridge University Press. Cambridge. UK, 2007. Translated from the German by Larry L. Schumaker.

[7] D. BRAESS, An a posteriori error estimate and a comparison theorem for the nonconforming PI element. Calcolo. 46 (2009), pp. 149-155.

[8] J. H. Bramble. R. D. Lazarov. And J. E. Pasciak. A least-squares approach based on a discrete minus one inner product for first onder systems, Math. Comp., 66 (1997), pp. 935955.

[9] J. BRANDTS. Y. (HEN. AND J. YANG, A note on least-squares mixed finite elements in relation to standard and mixed finite elements. IMA J. Numer. Anal., 26 (2006), pp. 779-789.

[10] S. C. BRENNER. Two-level additive Schwarz preconditioners for nonconforming finite element methods, Math. Comp., 65 (1996), pp. 897-921.

[11] S. C. BRENNER, Convergence of nonconforming multigrid methods without full elliptic regularity. Math. Comp., 68 (1999), pp. 25-53.

[12] S. C. Brenner, Poincaré-Friedrichs inequalities for piecewise $H^{1}$ functions, SIAM J. Numer. Anal., 41 (2003), pp. 306-324.

[13] C. Carstensen. R. H. W. Hoppe. C. Löbhard. and M. Eigel, A review of unified a posterioti finite element error control, Numer. Math. Theory Methods Appl., 5 (2012), pp. $509-558$.

(14) C. CARSTENSEN AND H. RABUS, An optimal adaptive mixed finite element method. Math. Comp. 80 (2011), pp. 649-667.

[15] J. M. Cascon. Ch. KReuzer. R. H. Nochetto. And K. G. Siebert, Quasi-optimal convergence nate for an adaptive finite element method, SIAM J. Numer. Anal., 46 (2008), pp. $2524-2550$.

[16] P. Castillo. B. Cockburn. 1. Perugia. and D. Schötzau, An a priori error analysis of the local discontinuous Galerkin method for elliptic problems, SIAM J. Numer. Anal., 38 (2000), pp. $1676-1706$.

(17) L. C.HEN. M. Holst. AND J. XU, Convergence and optimality of adaptive mixed finite element methods. Math. Comp., 78 (2009). pp. 35-53.

[18] P. G. CiARlet AND J. L. Lions, EDS., Handbook of Numerical Analysis, Vol. VII. Handbook of Numerical Analysis. VII, North-Holland. Amsterdam, 2000.

[19] B. CockbURn AND C.-W. ShU, The lacal discontinuous Galerkin method for time-dependent convection-diffusion systems, SIAM J. Numer. Anal., 35 (1998), pp. 2440-2463.

[20] R. Courant, On a method for the solution of boundary-value problems. in Theodore von Kármán Anniversary Volume, California Institute of Technology, Pasadena, CA, 1941. pp. $189-194$.

[21] M. Crouzeix and P.-A. Raviart, Conforming and nonconforming finite element methods for solving the stationary Stokes equations. I, Rev. Française Automat. Informat. Recherche Opérationnelle Sér. Rouge, 7 (1973), pp. 33-75.

[22] J. Doliglas. JR.. AND T. DUPONT, Interior penalty procedures for elliptic and parabolic Galerkin methods, in Computing Methods in Applied Sciences (Second Internat. Sympos.. Versailles. 1975), Lecture Notes in Phys. 58, Springer, Berlin, 1976, pp. 207-216.

[23] L. Fezoli. Fatima. S. Lanteri. B. Larroliturou, and C. Olivier, Resolution numetique des equations de Navier-Stokes pour un fluide compressible en maillage triangulaire, Research Report RR-1033. INRIA. Urbana. IL. 1989.

[24] T. GuDI, A new error analysis for discontinuous finite element methods for linear elliptic problems, Math. Comp., 79 (2010), pp. 2169-2189. 
[25] P. Houston. D. SchötZau. AND T. P. Wihler, Enengy notm a posteriori errat estimation of hp-adaptive discontinuous Galerkin methods for elliptic problems, Math. Models Methods Appl. Sci., 17 (2007), pp. 33-62.

[26] O. A. KaRAkashian And F. PASCal, A posteriori error estimates for a discontinuous Galerkin approximation of second-onder elliptic problems, SIAM J. Numer. Anal,, 41 (2003), pp. 2374-2399.

[27] J. Ku, Weak coupling of solutions of first-order least-squares method, Math. Cornp., 77 (2008), pp. 1323-1332.

[28] L. D. MARINI, An inexpensive method for the evaluation of the solution of the lowest order Raviart-Thomas mixed method, SIAM J. Numer. Anal., 22 (1985), pp. 493-496.

[29] H. RABUS, A natural adaptive nonconforming FEM of quasi-optimal complexity, Comput. Methods Appl. Math., 10 (2010), pp. 315-325.

[30] P.-A. Raviart And J. M. Thomas, A mixed finite element method for 2nd order elliptic problems, in Mathematical Aspects of Finite Element Methods (Proc. Conf., Consiglio Naz. delle Ricerche (C.N.R.), Rome, 1975), Lecture Notes in Math. 606, Springer, Berlin, 1977, pp. 292-315.

[31] B. Rivière, M. F. Wheeler, and V. Girault, A priori etrot estimates for finite element methods based on discontinuous approximation spaces for elliptic problems, SIAM J. Numer. Anal., 39 (2001). pp. 902-931.

[32] R. Verfürth, A Review of A Posteriori Error Estimation and Adaptive Mesh-refinement Techniques, Advances in Numerical Mathematics. Wiley-Teubner, Stuttgart, Germany, 1996. 